\title{
Multiresolution Analysis for Early Diagnosis of Alzheimer's Disease
}

\author{
Genevieve Jacques ${ }^{1}$, Jennifer L. Frymiare ${ }^{2}$, John Kounios ${ }^{2}$, Christopher Clark ${ }^{3}$, and Robi Polikar ${ }^{1}$ \\ ${ }^{1}$ Department of Electrical and Computer Engineering, Rowan University, Glassboro, New Jersey, USA \\ ${ }^{2}$ Department of Psychology, Drexel University, Philadelphia, Pennsylvania, USA \\ ${ }^{3}$ Department of Neurology, University of Pennsylvania, Philadelphia, Pennsylvania, USA
}

\begin{abstract}
Early diagnosis of Alzheimer's disease is a major concern due to large portions of the elderly population it affects and the lack of a standard and effective diagnosis procedure that is available to community healthcare providers. Several studies have been performed using wavelets or other signal processing methods to analyze EEG signals in an attempt to find a biomarker for Alzheimer's disease, which showed varying degrees of success. To date, in part due to lack of a large study cohort, the results of these studies remain largely inconclusive. In this paper, we describe a new effort using multiresolution wavelet analysis on event related potentials of the EEG to investigate whether such a link can be established. Several factors sets this study apart from similar prior efforts: We use a larger cohort, compare different mother wavelets, rather then using one generic wavelet, and most importantly, we specifically target early diagnosis of the disease. Our multi-year effort will include a total of 80 patients, whose ERPs will be analyzed with several different wavelets and automated classifiers. We present some preliminary, yet very promising, results on analysis of EEGs of the first 28 patients analyzed thus far using two types of wavelets.
\end{abstract}

Keywords-Alzheimer's disease, automated classification, multiresolution analysis, neural networks, oddball paradigm.

\section{INTRODUCTION}

Alzheimer's disease (AD) affects an estimated 4 million Americans, making it a major public health concern. The positive predictive value of clinical diagnosis is around $93 \%$ at University research clinics, however, most patients are evaluated by community healthcare providers, where the expertise and the accuracy of disease specific dementia diagnoses remains uncertain. In fact, a recent study reported that despite the advantage of longitudinal follow up, a group of Health Maintenance Organization-based physicians had a sensitivity of $83 \%$, specificity of $55 \%$ and an overall accuracy of $75 \%$ for the clinical diagnosis of $\mathrm{AD}$ [1]. These are the numbers we wish to match or exceed. Considering that community clinics constitute the first line of intervention, particularly at the early stages of their disease, an effective and objective tool for early diagnosis of the disease is of significant importance. To have a meaningful impact on healthcare, the diagnosis procedures must be inexpensive, non-invasive and available to community physicians.

Several biomarkers have been linked to AD, such as the cerebrospinal fluid tau $(\mathrm{CSF}-\tau), \beta$-amyloid, urine F2isoprostane, brain atrophy and volume loss detected by magnetic resonance imaging, or detection of reduced $\mathrm{N}$ acetyl aspartate by magnetic resonance spectroscopy. However, none of these methods has proven to be conclusive, and even if they were, they remain primarily university based research tools. Consequently, there is significant need for a clinically useful, accurate, non-invasive, cost-effective and automated procedure for early diagnosis of the AD. One such tool that is potentially feasible for AD diagnosis is the electroencephalogram (EEG).

EEG analysis has not traditionally been part of general physicians routine evaluation for $\mathrm{AD}$ diagnosis, in part due to difficulties in separating EEG changes that could be attributed to $\mathrm{AD}$ from those due to normal aging, coexisting medical illnesses, the level of anxiety or drowsiness during the recording and possible medication effects. An alternative EEG based technique targets the changes due to mental impairment by analyzing scalp recordings of auditory event related (evoked) potentials (ERP). This protocol uses the oddball paradigm in which subjects are instructed to push a button when they hear an occasionally occurring $2 \mathrm{kHz}$ tone (the oddball tone), presented randomly within a series of frequently occurring $1 \mathrm{kHz}$ tones. The ERPs then show a positive peak (P3 or P300) with an approximate latency of $300 \mathrm{~ms}$. after the stimulus. The distinguishing feature of the P300 is that it only occurs in response to the oddball stimulus. Changes in the amplitude and latency of ERPs are altered by neurological disorders affecting the temporalparietal regions of the brain [2]. This includes AD where the average stimulus to P300 latency is prolonged and the amplitude decreased compared to controls. Furthermore, Polich et al. have reported that latency and the amplitude of the P300 were altered in patients with early AD when compared to elderly control subjects $[3,4]$.

Traditional ERP analysis is performed in the time domain using the amplitude and latency of the averaged P300s. However, conventional time domain analysis reveals only a fraction of the information, since the ERP is a time and frequency varying signal, reflecting the sum of many neural events that occur in response to the stimulus. Although it has been around for about 15 years, studies applying timefrequency analysis techniques, such as wavelets, to ERPs have just started appearing in the literature. These studies seek to decompose an ERP in the time-frequency plane in order to characterize its functionally relevant components. Initial studies have shown that the ERPs in general, and the P300 component in particular, consists of superposition of 
multiple functional components, where these components extend for different, yet overlapping, time intervals in different frequency bands [5]. For example, in a study of 10 healthy young adults Demiralp et al. were able to generate a discriminant function using only seven DWT coefficients of the oddball ERP that could correctly identify $78 \%$ of the stimulus targets and $81 \%$ of the non-target tones [6].

The method explained in this paper combines established electroencephalography (EEG) analysis, multiresolution wavelet analysis (MWA) and automated classification techniques, to detect the earliest neurodegenerative changes of AD. MWA is a promising method for concurrent temporal and spectral analysis of non-stationary ERPs, while artificial neural network (ANN) based classifiers offer a powerful automated classification tool for generating complex decision boundaries. Our objective is to analyze the entire ERP using MWA followed by an ANN classification, not just the P300, with the hypothesis that there are additional features or biomarkers in an ERP other then the P300 that may indicate the early presence of the AD. We compare two different types of wavelets on the first 28 of the 80 patients scheduled for this study. We are interested in determining the sensitivity, specificity, and positive predictive value of the above-mentioned system, trained with wavelet coefficients of ERPs, to categorize elderly individuals as either patients with $\mathrm{AD}$ or cognitively normal subjects.

\section{METHODOLOGY}

\section{A. Test Subjects and Clinical Evaluation}

This study will include a total of 80 subjects, 50 of whom will be used to train the automated classification system, and the remaining 30 will be used to evaluate and validate the system's performance on previously unseen signals. Half of these subjects constitute the cognitively normal cohort, whereas the other half constitutes the AD cohort. Thirty-two elderly test subjects, 14 diagnosed with probable $\mathrm{AD}$ and 18 cognitively normal controls, have been recruited thus far in the initial phase of this study. All subjects, control or with probable AD, are recruited from elderly patients, over 60 years of age. The subjects were verified to be free of any evidence of central nervous system neurological disease (e.g. stroke, multiple sclerosis, Parkinson's disease, etc.) by history or by exam. The use of sedative, anxiolytic or antidepressant medications was not permitted within 48 hours of data collection. The two groups were defined by the following criteria: Cognitively normal subjects: (i) Clinical Dementia Rating $(\mathrm{CDR})=0$; (ii) Mini-Mental Scores (MMS) $\geq 24$; (iii) no indication of functional or cognitive decline during the two years prior to enrollment based on a detailed interview with the subject's knowledgeable informant or two previous annual clinical assessments. AD subjects: (i) $\mathrm{CDR} \geq 0.50$; (ii) $\mathrm{MMS}<<24$; (iii) presence of functional cognitive decline over the previous 12 months based on detailed interview with a knowledgeable informant; (iv) satisfaction of NINCDS-ADRDA (National Institute of Neuro- logical and Communicative Disorders and Stroke - Alzheimer's Disease and Related Disorders Association) criteria for probable $\mathrm{AD}[7]$.

All subjects receive a through medical history and neurological exam at the University of Pennsylvania's Memory Disorders Clinic, in Philadelphia. Key demographic and medical items, including current medications (prescription, over-the-counter, and complementary alternative medications) are entered into their case binder and Access ${ }^{\mathrm{TM}}$ database. The evaluation includes standardized assessments for overall impairment, functional impairment, extrapyramidal signs, behavioral changes and depression as listed below: CDR score; Dementia Severity Rating Scale; Penn Behavioral Ratings Scale; Geriatric Depression Scale; Verbal Fluency test; Boston Naming Test; Mini-Mental State exam; Word List Memory; Constructional Praxis; Word list recall; Word List Recognition; Logical Memory I \& II; Clock draw \& copy; Digit Symbol Substitution; Hand tasks; Praxis Recall; and Praxis Recognition.

\section{B. Acquisition of Event Related Potentials}

The ERPs were obtained using an auditory oddball paradigm while the subjects were comfortably seated in a specially designated room. The protocol originally described by [2], with slight modifications was used in this study. Binaural audiometric thresholds were determined for each subject using a $1000 \mathrm{~Hz}$ tone. The evoked response stimulus was presented to both ears using stereo speakers at an amplitude comfortable for their hearing level.

The stimulus consisted of tone bursts $100 \mathrm{~ms}$ in duration, including $5 \mathrm{~ms}$ inset and offset envelopes. Tones of 1000 and $2000 \mathrm{~Hz}$ were presented in a random sequence with the tones occurring in $65 \%$ and $20 \%$ of the trials respectively. The remaining $15 \%$ of the trials consisted of novel sounds presented randomly. These included 60 unique environmental sounds that were recorded digitally and edited to a $200 \mathrm{~ms}$ duration. A total of 1000 stimuli, including frequent $1000 \mathrm{~Hz}$ $(n=650)$, infrequent $2000 \mathrm{~Hz}$ tones $(n=200)$ and novel sounds $(n=150)$ were delivered to each subject with an interstimulus interval of 1.0-1.3 seconds. The subjects were instructed to press a button each time they heard the $2000 \mathrm{~Hz}$ tone. With frequent breaks (e.g. approximately three minutes of rest every five minutes), the data collection process lasted about 30 minutes per subject with each session proceeded by a 1 minute practice session without the novel sounds.

The ERPs were recorded from 19 tin electrodes embedded in a plastic cap, using linked mastoids as reference. The electrode impedances were kept below $20 \mathrm{k} \Omega$ to yield a good signal with the high-impedance MANSCAN amplifier system used in the study. Artifactual recordings were identified and rejected by the EEG technician. The remaining scalp potentials were amplified, digitized at 250Hz/channel (19 channels) and stored. The ERPs that were validated by the EEG technician were preprocessed using appropriate lowpass filtering techniques and averaging. The averaging pro- 
tocol involved averaging 30 85 recordings per patient yielding 1 3 recordings per patient. The inherent variability of ERPs ensures that no two recordings are ever identical and therefore separate averages were all included in the analysis to provide additional data. All averages have been notched filtered at $59-61 \mathrm{~Hz}$ and baselined with the prestimulus interval. Four of the original 32 subjects were unable to complete the study due to various reasons, and hence their recordings were excluded from further analysis. All multiple averages from the 28 remaining subjects, a total of 75 recordings, 26 from $\mathrm{AD}$ and 49 from normal controls, were therefore analyzed.

\section{Multiresolution Wavelet Analysis}

Time localizations of spectral components can be obtained by multiresolution wavelet analysis, as this method provides the time-frequency representation of the signal. Among many time-frequency representations, the discrete wavelet transform (DWT), has become increasingly popular due to its ability to solve a diverse set of problems, including data compression, biomedical signal analysis, feature extraction, noise suppression, density estimation, and function approximation, with modest computational expense. For brevity, we only describe the main points here and refer the interested readers to many excellent references listed at [8].

The DWT analyzes the signal at different frequency bands with different resolutions through the decomposition of the signal (multiresolution analysis). The DWT utilizes two sets of functions: scaling functions and wavelet functions, each associated with lowpass and highpass filters, respectively. Decomposition of the signal into different frequency bands is accomplished by successive highpass and lowpass filtering of the time domain signal.

The original time domain signal $\mathbf{x}(\mathbf{t})$ sampled at 250 samples/ sec. creates the discrete time signal, $\boldsymbol{x}[\boldsymbol{n}]$ which is passed through a halfband highpass filter, $\boldsymbol{g}[\boldsymbol{n}]$, and a lowpass filter, $\boldsymbol{h}[\boldsymbol{n}]$. In terms of angular frequency, the highest frequency in the original signal is $\pi$, corresponding to the linear frequency of $125 \mathrm{~Hz}$. According to Nyquist's rule, half the samples can be removed after the filtering, since the highest frequency in the signal is now $\pi / 2$ radians. Therefore every other sample in the signal can be discarded. This is one level of decomposition and can be expressed as follows:

$$
\begin{aligned}
& y_{\text {high }}[k]=\sum_{n} x[n] \cdot g[2 k-n] \\
& y_{\text {low }}[k]=\sum_{n} x[n] \cdot h[2 k-n]
\end{aligned}
$$

where $\boldsymbol{y}_{\text {high }}[\boldsymbol{k}]$ and $\boldsymbol{y}_{\text {low }}[\boldsymbol{k}]$ are the outputs of the highpass and lowpass filters, respectively, after the subsampling. This procedure, called subband coding, is repeated for further decomposition. At each level, the filtering procedure results in half the time resolution and double the frequency resolution. Therefore, each level of decomposition analyzes the signal at different frequency ranges and different resolutions. Fig.
1 illustrates this procedure, where $\boldsymbol{x}[\boldsymbol{n}]$ is the original signal to be decomposed, and $\boldsymbol{h}[\boldsymbol{n}]$ and $\boldsymbol{g}[\boldsymbol{n}]$ are impulse responses of lowpass and highpass filters, respectively. The bandwidth of the signal at every level is marked on the figure as " $\mathrm{B}$ ".

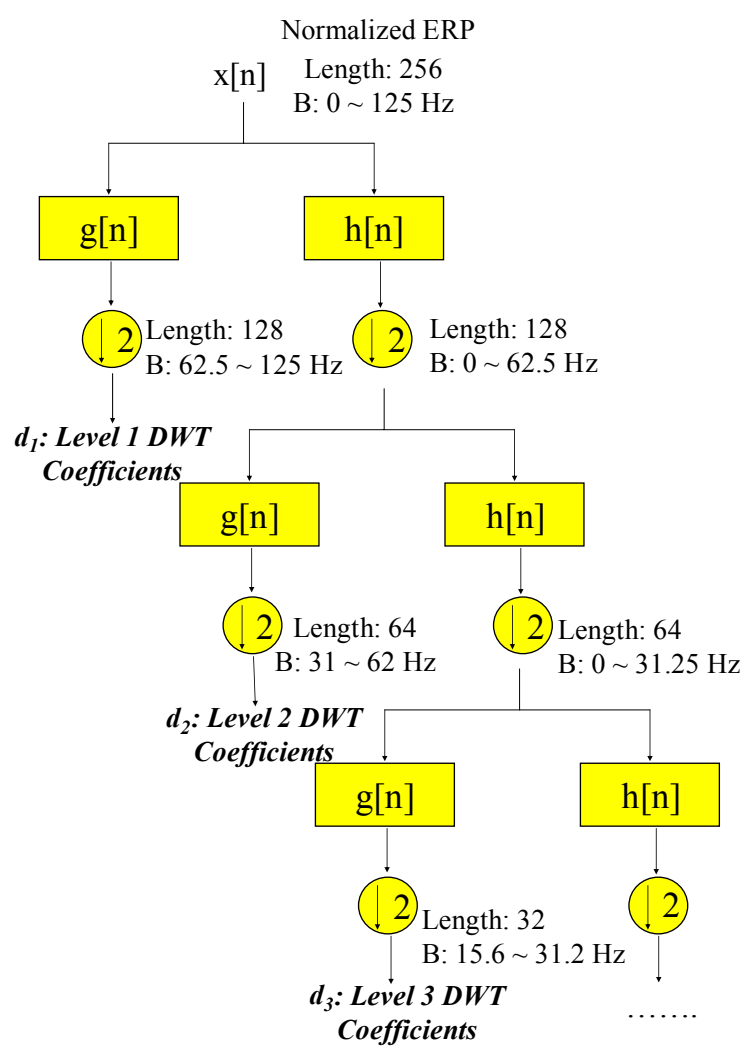

Fig.1. DWT Subband coding algorithm from

\section{Automated Classification using Neural Networks}

A standard procedure in automated classification applications is to first obtain the distinctive features of the signals to be identified, followed by training a neural network with a training data consisting of those features. The trained network is then evaluated on data not seen by the network during the training. In this study, The DWT coefficients, timefrequency space features of the ERP, are used to train a single hidden layer multiplayer perceptron (MLP) type neural network using backpropagation training algorithm [9]. The MLP is a well established technique, and its implementation as well as its training algorithms can be found in many references such as [9].

\section{RESULtS}

Two types of wavelets were used: Daubechies 4 (db4) wavelet and Quadratic b-spline wavelet, both of which have been used in prior efforts $[5,10]$. The number of coefficients varied based on the wavelet, 121 for b-spline and 64 for $\mathrm{db} 4$, though they were chosen to include all frequencies below $20 \mathrm{~Hz}$, where most of the ERP information is known to reside. The decomposition of one of the normal controls is shown in Fig. 2, where $d_{1} \sim d_{7}$ indicate the detail coeffi- 
cients and $a_{7}$ is the remaining signal after all the details are removed from the signal (hence a low frequency signal). Note that the amplitudes of the coefficients decrease at lower levels, and hence those levels can be discarded. Frequencies below $20 \mathrm{~Hz}$ include levels 5 and above according to 250 samples/sec sampling rate, and hence these coefficients were retained for neural network training. We also point to the positive peak in $d_{5}$ around $400 \sim 500 \mathrm{~ms}$, which probably correspond to a late P300.

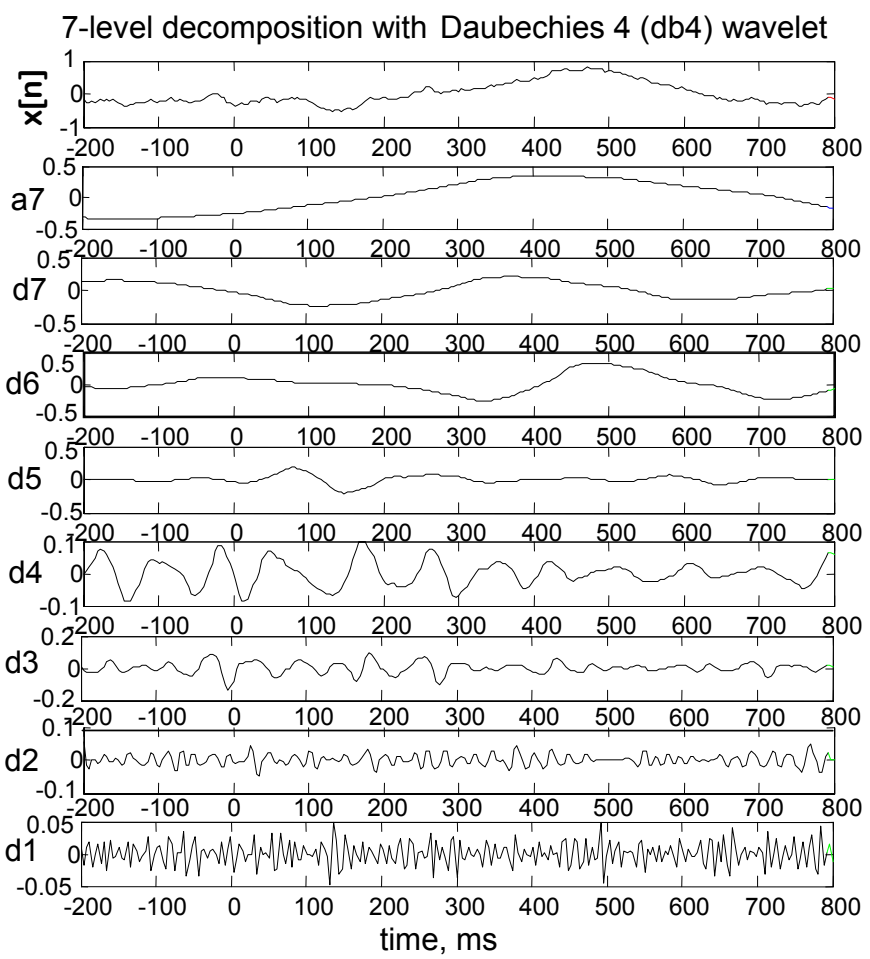

Fig. 2. DWT decomposition of a control ERP using db-4 wavelet

While 19 channels of EEGs were recorded, we have thus far analyzed the Pz electrode, the channel that is most commonly used in oddball paradigm analysis. Several simulations were conducted using different training and test datasets. In this initial effort, 38 (25 normal, $13 \mathrm{AD}$ ) of the 75 recordings were randomly selected for training, and remaining 37 ( 24 normal, 13 AD) were used to evaluate the generalization performance of the classifier. Over ten random trials, the db4 wavelet was able to reach an average overall performance of $84.1 \pm 0.6 \%$ (32/37) and b-splines were able to reach $82.4 \% \pm 1.0 \%(31 / 37)$. The network parameters were 25 hidden layer nodes with an error goal of 0.05 for $\mathrm{db} 4$, and 0.01 for the b-splines. The sensitivities were $77 \%$ and $54 \%$, the specificities were $92 \%$ and $100 \%$, and the positive predictive values were $83.3 \%$ and $100 \%$, for $\mathrm{db} 4$ and b-splines wavelets, respectively. We have also tried using two-third of the data for training and one-third for testing. The generalization performance in this case was $88 \pm$ $2.4 \%$ for $\mathrm{db} 4$ wavelet and $88 \pm 1.2 \%$ for the b-splines.

\section{DISCUSSIONS \& CONCLUSIONS}

While these results are very preliminary and based on a very limited number of patients, they do support the feasibility of this approach as a tool for classifying ERPs. Based on these results we make the following observations and conclusions: (i) Using wavelet analysis to extract features of the ERPs, followed by an automated neural network classifier appears to be a feasible approach for early diagnosis of $\mathrm{AD}$; (ii) the type of wavelet may not be too critical, as both extract much of the same information, however, the approach can be optimized for a specific wavelet; (iii) we have also tried several network architectures and error goals for the MLP, and the approach is remarkably invariant to minor changes in these parameters; (iv) it comes as no surprise that having additional training data certainly helps improve the generalization performance. Therefore the approach should provide a stable and effective algorithm once the remaining patients are recruited and their signals are integrated into the knowledge base of the classifier.

Besides recruiting the remaining cohort, future work will include investigating other mother wavelets, analyzing additional channels other then $\mathrm{Pz}$, and a more rigorous evaluation of the algorithm using several levels of cross-validation.

\section{ACKNOWLEDGMENT}

This work is supported by National Institute on Aging of the National Institutes of Health under grant number P30 AG10124 - R01 AG022272.

\section{REFERENCES}

[1] A. Lim, D. Tsuang, et al." Clinico-neuropathological correlation of Alzheimer's disease in a community-based case series," J American Geriatrics Society vol. 47, no. 5, pp. 564-569, 1999.

[2] S. Yamaguchi, H. Tsuchiya, S. Yamagata, G. Toyoda, S. Kobayashi, "Event-related brain potentials in response to novel sounds in dementia," Clinical. Neurophysiology, vol. 112, no. 2, pp. 195-203, 2002.

[3] J. Polich, C. Ladish, F. Bloom, "P300 assessment of early Alzheimer's disease," EEG \& Clin. Neurophys. vol. 77, no. 3, pp. 179-189, 1990.

[4] J. Polich, "P300 in clinical applications, ” In Electroencephalography, E. Niedermeyer, F. Lopez Da Silva, Ed. Philadelphia: Williams and Wilkins, 1999, pp. 1073-1091.

[5] T. Demiralp, A. Ademoglu, "Decomposition of event-related brain potentials into multiple functional components using wavelet transform," Clinical Electroencephalography vol. 32, no. 3, pp. 122-138, 2001.

[6] T. Demiralp et al. "Analysis of functional components of P300 by wavelet transform, ” Proc. of IEEE Eng. in Med. \& Bio. Conf., vol. 20, no.4, pp. 1992-1995, 1998.

[7] G. McKhann, et al., "Clinical diagnosis of Alzheimer's disease: report of the NINCDS-ADRDA Work Group to Dept. of HHS Task Force on Alzheimer's Disease," Neurology, vol. 34, pp. 939-944, 1984.

[8] M. Unser, editor, Gallery at wavelet.org, 04/10/2004, Available at: http://www.wavelet.org/phpBB2/gallery.php

[9] S. Haykin, Neural Networks, A Comprehensive Foundation, Upper Saddle River, NJ: Prentice Hall, 1998.

[10] R. Polikar, F. Keinert, M.H. Greer, "Wavelet analysis of ERPs for early diagnosis of Alzheimer's disease," in Wavelets in Signal and Image Analysis, From Theory to Practice (ed. A. Petrosian and F.G. Meyer), pp. 453-478, Boston, MA: Kluwer Academic Publishers, 2001. 